\title{
Repurposing Screen Identifies Unconventional Drugs With Activity Against Multidrug Resistant Acinetobacter baumannii
}

\author{
Yu-Shan Cheng ${ }^{1}$, Wei Sun ${ }^{1}$, Miao Xu ${ }^{1}$, Min Shen ${ }^{1}$, Mozna Khraiwesh ${ }^{2}$, Richard J. Sciotti ${ }^{2}$ \\ and Wei Zheng ${ }^{1 *}$
}

${ }^{1}$ National Center for Advancing Translational Sciences, National Institutes of Health, Bethesda, MD, United States,

${ }^{2}$ Experimental Therapeutics Branch, Walter Reed Army Institute of Research, Silver Spring, MD, United States

\section{OPEN ACCESS}

Edited by:

Rodolfo García-Contreras, National Autonomous University of

Mexico, Mexico

Reviewed by:

Attilio Vittorio Vargiu,

Università degli Studi di Cagliari, Italy

Bernardo Franco,

Universidad de Guanajuato, Mexico

*Correspondence: Wei Zheng

wzheng@mail.nih.gov

Specialty section

This article was submitted to

Clinical Microbiology,

a section of the journal

Frontiers in Cellular and Infection

Microbiology

Received: 14 August 2018 Accepted: 10 December 2018

Published: 04 January 2019

Citation:

Cheng Y-S, Sun W, Xu M, Shen M, Khraiwesh M, Sciotti RJ and Zheng $W$ (2019) Repurposing Screen Identifies

Unconventional Drugs With Activity Against Multidrug Resistant

Acinetobacter baumannii.

Front. Cell. Infect. Microbiol. 8:438.

doi: 10.3389/fcimb.2018.00438
Antibiotic-resistant nosocomial infections are an emerging public health issue; carbapenem-resistant gram-negative bacteria such as Acinetobacter baumannii are among the pathogens against which new therapeutic agents are desperately needed. Drug repurposing has recently emerged as an alternative approach to rapidly identifying effective drugs and drug combinations to combat drug resistant bacteria. We performed a drug repurposing screen against a highly virulent, multidrug resistant, Acinetobacter baumannii strain AB5075. This strain, isolated from a patient, is resistant to 25 first-line antibiotics for gram-negative bacteria. A compound screen using a bacterial growth assay led to identification and confirmation of 43 active compounds. Among these confirmed compounds, seven are approved drugs or pharmacologically active compounds for non-antimicrobial indications. Three of these drugs, 5-fluorouracil, fluspirilene, and Bay 11-7082 resensitized strain AB5075 to azithromycin and colistin in a two-drug combination format. The approach using a drug repurposing screen with a pathogen sample isolated from a patient and a high throughput bacterial growth assay led to the successful identification of new drug combinations to overcome a multidrug resistant bacterial infection.

Keywords: Acinetobacter baumannii, multidrug resistance, drug repositioning, drug repurposing screen, synergistic drug combination, nosocomial infections, non-antimicrobial drugs

\section{INTRODUCTION}

The emergence and dissemination of drug-resistant bacterial infections are a public health issue. Acinetobacter baumannii is one of the major causes for the nosocomial infections in critically ill patients. Treatment of Acinetobacter baumannii can be extremely difficult, especially for the carbapenem resistant strains. Colistin and tigecycline are the last resorts for carbapenem resistant Acinetobacter baumannii. However, colistin and tigecycline resistant strains have been reported worldwide (Deng et al., 2014; Oikonomou et al., 2015). In light of the rapid expansion of imipenem resistance in 35 countries, increasing from 24 to $74 \%$ in just 11 years (Xie et al., 2018), the development of novel drugs to combat Acinetobacter baumannii infections is an urgent need.

Antibiotic development mainly relies on two strategies, a target-based approach and isolation of bioactive secondary metabolites from microorganisms (Demain, 1999; Marinelli, 2009). New antibiotic drug development is a long-term process; for example, a target-based method takes time to go through the steps of target selection, lead discovery and optimization, preclinical 
development, then clinical trials before the FDA gives approval for marketing for a new indication. Drug repurposing and drug combinations have emerged as promising alternative approaches to provide novel therapeutic options for multidrug resistant bacteria (Zheng et al., 2018). Drug repurposing of approved drugs bypasses the need for novel molecules in nature or from a synthetic chemical library, and alleviates the need for preclinical development and phase I clinical trials as the data for preclinical experiments, human pharmacokinetics, and drug safety are already established. Thus, drug repurposing accelerates the drug development process and reduces the development costs. In addition, drug combination therapy with a synergistic effect of two or three drugs in combination can overcome drug resistance by inhibiting multiple targets and reducing occurrence of further drug resistance.

A multidrug resistant Acinetobacter baumannii clinical isolate, Acinetobacter baumannii 5075 (AB5075) (Jacobs et al., 2014), was used in this study. Strain AB5075 was first isolated from the osteomyelitis of a patient's tibia bone in 2008. The detailed genomic analysis of AB5075 in 2015 unveiled some antibiotic resistant mechanisms (Gallagher et al., 2015). Briefly, AB5075 carries 133 genes that likely cause resistance to broad-spectrum $\beta$-lactams (for example, penicillins, cephalosporins, and carbapenems), aminoglycosides, chloramphenicol, quinolones, tetracycline, trimethoprim, sulfonamides, macrolides, and other toxic agents. We report here the identification of seven non-antimicrobial drugs that suppressed AB5075 growth in vitro by a drug repurposing screen using the AB5075 strain. The results demonstrate the usefulness of a drug repurposing screen using patient derived pathogens. These newly identified compounds with inhibitory activities against multidrug resistant Acinetobacter baumannii can be further studied for use as new therapeutic agents.

\section{MATERIALS AND METHODS}

\section{Materials}

Tigecycline was obtained from Chem-Impex International (Wood Dale, IL, USA). Doripenem and ertapenem were acquired from Cayman Chemical (Ann Arbor, MI, USA) and TOKUE (Bellingham, WA, USA), respectively. Other chemicals were purchased from Sigma-Aldrich (St. Louis, MO, USA).

\section{Preparation of Bacterial Stock for High Throughput Screen}

Acinetobacter baumannii 5075 (AB5075) was obtained from Walter Reed Army Institute of Research. Individual colonies on agar plates were cultured in tryptic soy broth (TSB, Remel, Thermo Scientific, Waltham, MA, USA) at $37^{\circ} \mathrm{C}$. The bacterial cultures were mixed with sterile glycerol in a 9:1 ratio when the optical density at $600 \mathrm{~nm}\left(\mathrm{OD}_{600}\right)$ reached about $0.25-0.3$. Bacteria in $10 \%$ glycerol were stored in aliquots at $-80^{\circ} \mathrm{C}$.

\section{Bacterial Growth Experiments}

Bacteria were thawed from $-80^{\circ} \mathrm{C}$ and diluted to a desire initial density from $1: 200$ to $1: 1,000$ in TSB. Each bacterial culture was grown in $\mathrm{TSB}$ at $37^{\circ} \mathrm{C}, 5 \% \mathrm{CO}_{2}$ humidified atmosphere for $2-48 \mathrm{~h}$. Bacterial growth was monitored by measuring the $\mathrm{OD}_{600}$ in a PHERAstar plate reader (BMG Labtech, Cary, NC, USA).

\section{Compound Library}

A pharmacologically active compound library (LOPAC 1280) was purchased from Sigma-Aldrich (St. Louis, MO, USA). The NCATS Pharmaceutical Collection (NPC) of approved and investigational drug collection was generated in house (Huang et al., 2011). The NPC library consists of 2,816 small molecule compounds, $38.4 \%$ approved by U.S. FDA, $22.5 \%$ approved in the EU, Canada, or Japan, and $39.0 \%$ being used in clinical trials or as research compounds.

\section{Compound Screening and Validation}

A quantitative high throughput screen (qHTS) and confirmation assays were performed as previously described in a 1,536well format (Sun et al., 2016). Briefly, $2.5 \mu \mathrm{L}$ TSB was first loaded into each well of a black clear bottom microplate by a Multidrop Combi dispenser (Thermo Fisher Scientific, Waltham, MA, USA). An automated pintool station (WAKO Scientific Solutions, San Diego, CA) was then used to transfer $23 \mathrm{~nL}$ of compounds from compound plates into assay plates. For the primary screen, each compound was tested at four concentrations. Compound plates were prepared by an Evolution $\mathrm{P}^{3}$ system (PerkinElmer, Wellesley, MA) as described in a previous publication (Inglese et al., 2006). In the followup confirmation assays, dose-effects of the carefully selected compounds were examined as an intraplate 11-point, 3-fold dilution series. Each compound was tested in three biological replicates. Dimethyl sulfoxide (DMSO) served as a negative control and $46 \mu \mathrm{M}$ of rifampin was the positive control. Bacterial stock diluted in TSB at a ratio 1:500 was added at $2.5 \mu \mathrm{L} /$ well to make a 1:1000 dilution of initial inoculum density. The assay plates were incubated at $37^{\circ} \mathrm{C}$ for $20-22 \mathrm{~h}$ and placed onto a PHERAstar plate reader (BMG Labtech, Cary, NC, USA) to detect the bacterial growth in response to $\mathrm{OD}_{600}$.

\section{Two Drug Combination Assays}

In two drug combination assays, non-antimicrobial drugs (drug 1) resulting from the qHTS were tested in combination with an 11-concentration series of 25 standard treatment drugs (drug 2). The screen was conducted in the same procedure as for qHTS except the TSB media was mixed with drug 1 prior to loading into the assay plates. The final concentrations of drug 1 were one-fourth and one-eighth of the calculated halfmaximal inhibitory concentration $\left(\mathrm{IC}_{50}\right)$ values. The dose-effect curve of drug 2 as a single agent or with a fixed concentration of drug 1 were calculated. A significant synergistic response was defined as a three-fold decrease in $\mathrm{IC}_{50}$. Drug pairs that showed a synergistic response were further validated in a 96-well plate.

\section{Statistics and Data Analysis}

The qHTS analysis was designed internally to include three steps: normalization, pattern correction, and curve fitting. Raw plate 

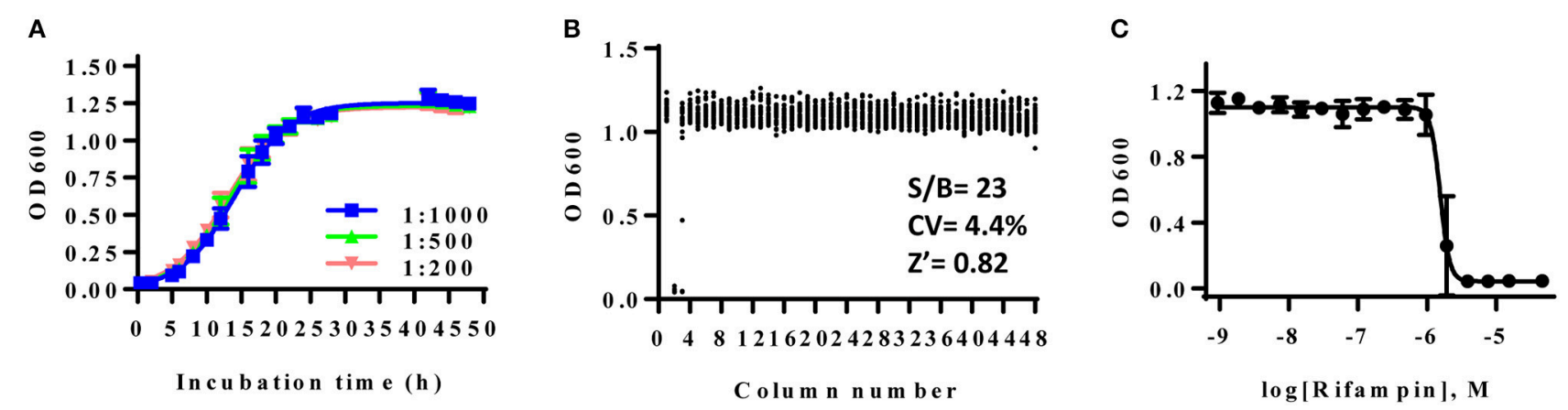

FIGURE 1 | (A) Growth curve of AB5075 in 1536-well plate. AB5075 stock solution was diluted to different starting ratios and incubated at $37^{\circ} \mathrm{C}$. Data points represent the mean, and the error bars represent the standard deviation (SD); $n=128$. (B) Scatter plot of the results from a DMSO plate screening. The wells in column 2 of the 1536-well assay plate contained $46 \mu \mathrm{M}$ rifampin as a positive control (0\% viability); the wells in column 3 contained varying doses of rifampin at $1: 3$ dilution. The wells in the rest of plate contained DMSO as a negative control (100\% viability). The signal-to-basal ratio (S/B) in this plate was 23 -fold, with a coefficient of variation (CV) of 4.4\%, and a Z' factor of 0.82. (C) Dose-response curves for rifampin from column 3 . The data points represent the mean, and the error bars represent the SD; $n=2$

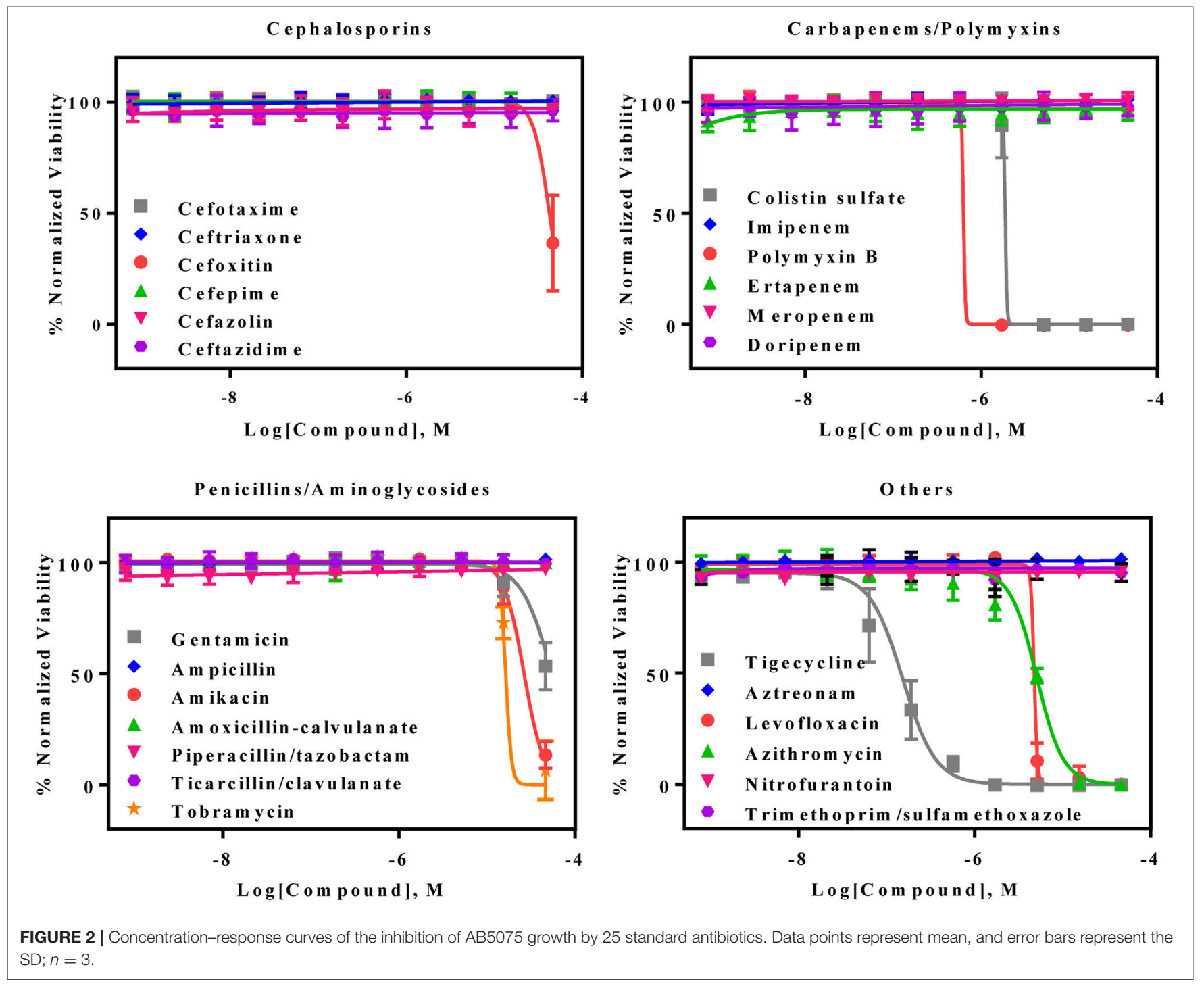


reads were first normalized to relative controls (such that DMSO alone was considered as $100 \%$ viability and $46 \mu \mathrm{M}$ rifampin as $0 \%$ viability) using the following equation: $\%$ normalized viability $=\left[\left(\mathrm{V}_{\text {compound }}-\mathrm{V}_{\text {positive }}\right) /\left(\mathrm{V}_{\text {DMSO }}-\mathrm{V}_{\text {positive }}\right)\right] \times 100$. Next the data underwent a pattern-correction based on information from a DMSO-alone plate. Interplate dose-response data of each compound was processed by a four-parameter Hill equation based grid algorithm to yield the $\mathrm{IC}_{50}$, the maximum response, and the curve class (Wang et al., 2010). IC $_{50}$ and IC 90 values in the confirmation experiments were calculated with Prism 7 software (GraphPad Software, Inc. San Diego, CA, USA).

\section{RESULTS}

\section{Assay Optimization for the High Throughput Bacterial Growth Assay}

The high throughput bacterial growth assay (Sun et al., 2016) were adapted to examine antibiotic susceptibility for AB5075.
The growth kinetics of AB5075 was monitored in a 1536well plate format to determine the optimal inoculum density and incubation time. Measurement of $\mathrm{OD}_{600}$ of assay plates, reflecting the bacterial growth rate, was recorded at various time points over $48 \mathrm{~h}$ (Figure 1A). A classical growth kinetic pattern was observed encompassing lag, exponential, and stationary phases; the growth reached stationary phase at $24 \mathrm{~h}$. As suggested by the antimicrobial susceptibility testing guideline for Acinetobacter spp. from the Clinical and Laboratory Standards Institute (CLSI), experimental endpoints were setting at $22-24 \mathrm{~h}$ for the following studies. All bacterial stock dilutions used for inoculum showed similar signal-to-basal ratios of $\sim 26$-fold. The dilution of 1:1,000 was chosen for use in further experiments.

The DMSO alone plate was used to account for well to well variation. Rifampin was selected to serve as the positive control compound in the experiments. The calculated signal-to-basal ratio was 23 -fold, the coefficient of variation $(\mathrm{CV})$ was $4.4 \%$, and the $\mathrm{Z}^{\prime}$ factor was 0.82 , indicating a robust assay for high

TABLE 1 | IC 50 and MIC data for standard care antibiotics against AB5075.

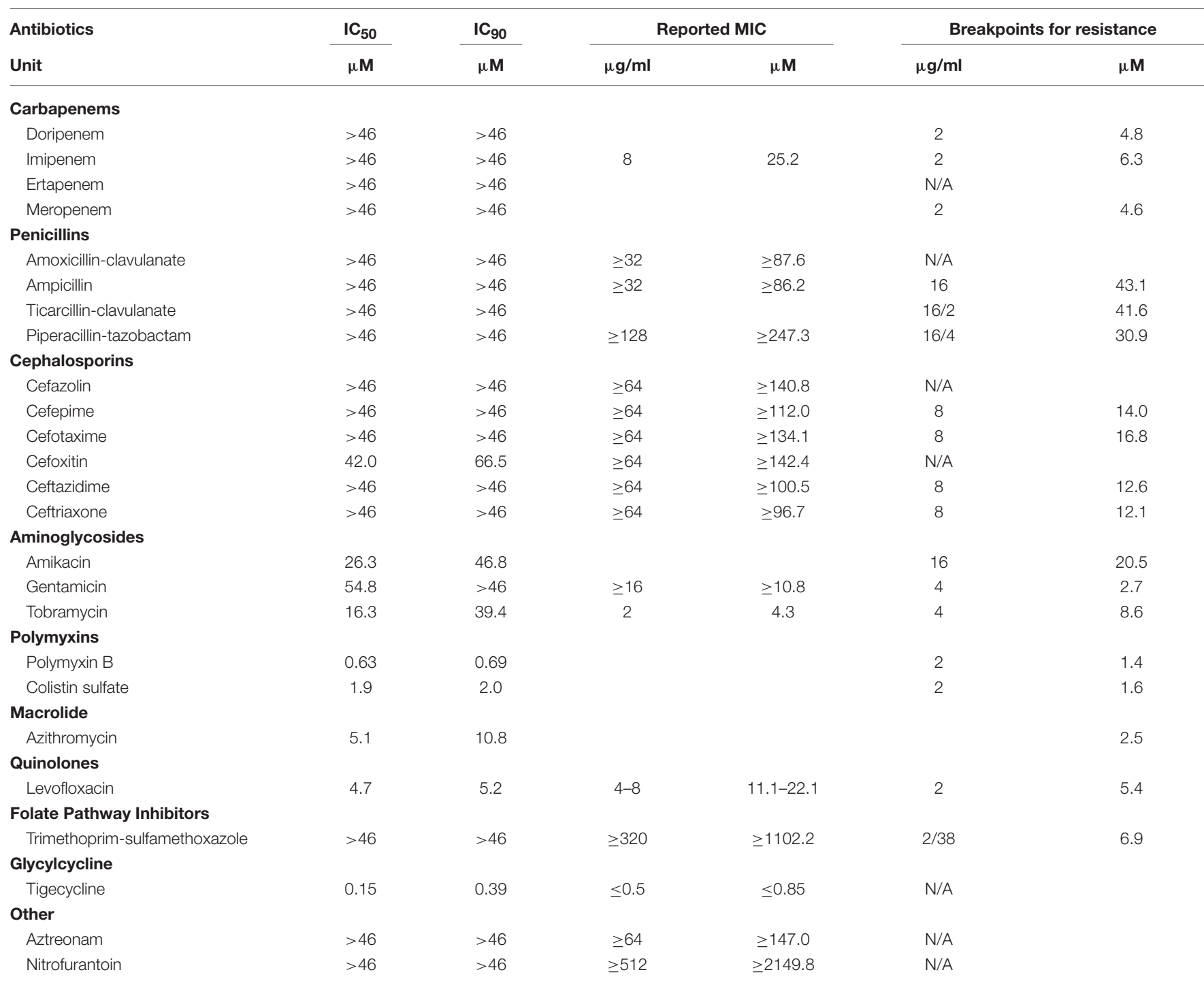


throughput screening (Figure 1B). The calculated $\mathrm{IC}_{50}$ value of rifampin against $\mathrm{AB} 5075$ was $0.40 \mu \mathrm{M}$ (Figure 1C).

\section{Activities of Standard Care Antibiotics Against AB5075 Strain}

The AB5075 growth assay was evaluated with a set of 25 antibiotics commonly used for infections by gram negative bacteria. Nine compounds showed concentration-dependent inhibition of AB5075 growth including tigecycline, two polymyxins (polymyxin B and colistin sulfate), levofloxacin, azithromycin, three aminoglycosides (amikacin, gentamicin, and tobramycin) and cefoxitin (Figure 2, Table 1). Among these active antibiotics, tigecycline was the most potent compound with an $\mathrm{IC}_{50}$ of $0.15 \mu \mathrm{M}$. The other 16 antibiotics were not active (i.e., $\mathrm{IC}_{50}>46 \mu \mathrm{M}$ ) against the multidrug resistant $\mathrm{AB} 5075$ strain.

\section{Repurposing Screen Using LOPAC and NPC Library}

The primary screen assessed 4,096 approved drugs and bioactive compounds to identify compounds inhibiting the growth of
AB5075 (Figure 3A). Each compound was tested at four concentrations $(4.1,9.2,20.6$, and $46 \mu \mathrm{M})$ in the primary screen. The primary hits were selected based on an $\mathrm{IC}_{50}$ below $30 \mu \mathrm{M}$ and an efficacy (maximum inhibition) $>70 \%$. Fifty-two compounds meeting these criteria were selected and retested, resulting in 43 confirmed compounds, an $83 \%$ confirmation rate (Table 2). These confirmed compounds include 30 antibacterial, 2 antifungal, 4 antiseptic, 3 antineoplastic, and 4 other agents (Figure 3B). Tetracycline and its analogs were the most potent compounds identified with $\mathrm{IC}_{50}$ values of $0.045-0.47 \mu \mathrm{M}$. Among the confirmed compounds, seven were drugs categorized as non-antimicrobial agents (Figure 3C, Table 3).

\section{Synergistic Drug Combinations of Newly Identified Non-antimicrobial Compounds and Standard Care Antibiotics}

A drug combination of a standard care antibiotic agent with a newly identified compound from the above confirmed compounds (non-antimicrobial agents) was screened using the same AB5075 growth assay. After additional testing of these individual compounds with 25 standard care antibiotic

\section{A}

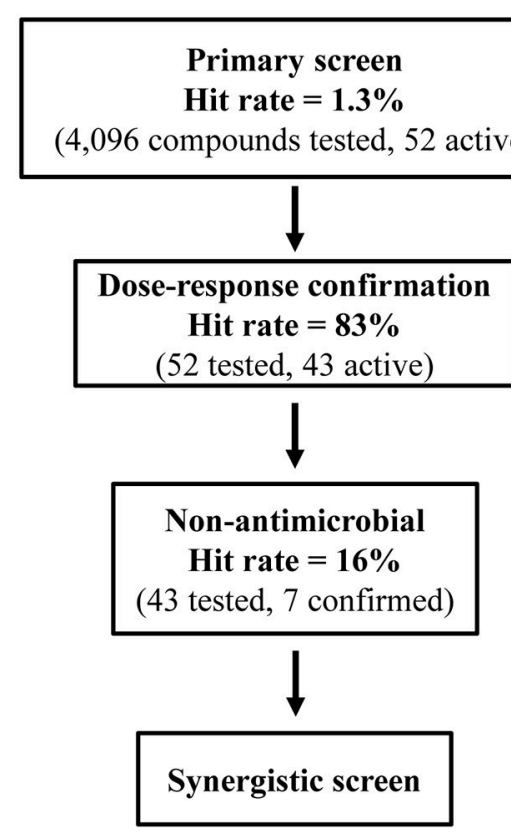

B

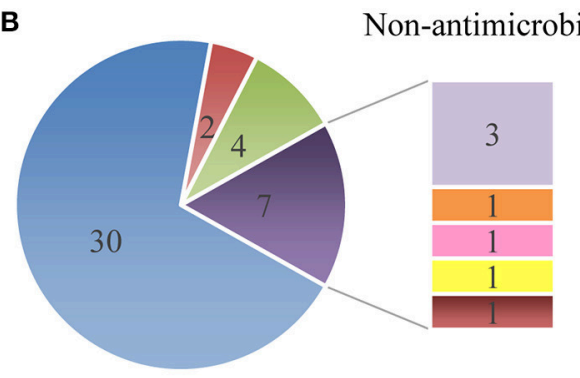

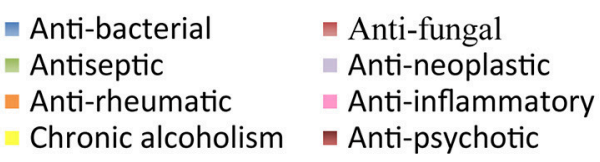


TABLE 2 | Antagonists identified in the qHTS.

\begin{tabular}{|c|c|c|c|}
\hline Drug name & $I_{50}(\mu \mathrm{M})$ & $\begin{array}{l}\text { Maximum } \\
\text { response }\end{array}$ & $\begin{array}{l}\text { Primary } \\
\text { action }\end{array}$ \\
\hline Doxycycline $\mathrm{HCl}$ & 0.045 & 109 & Antibacterial \\
\hline Minocycline $\mathrm{HCl}$ & 0.094 & 112 & Antibacterial \\
\hline Demeclocycline $\mathrm{HCl}$ & 0.13 & 94 & Antibacterial \\
\hline Methacycline $\mathrm{HCl}$ & 0.13 & 84 & Antibacterial \\
\hline Sancycline & 0.14 & 104 & Antibacterial \\
\hline Rifampicin & 0.45 & 100 & Antibacterial \\
\hline Tetracycline $\mathrm{HCl}$ & 0.47 & 91 & Antibacterial \\
\hline Thimerosal & 0.53 & 92 & $\begin{array}{l}\text { Antiseptic and } \\
\text { germicides }\end{array}$ \\
\hline Triclosan & 0.93 & 90 & Antibacterial \\
\hline Auranofin & 1.09 & 106 & Antirheumatic \\
\hline Gatifloxacin & 1.37 & 105 & Antibacterial \\
\hline Sitafloxacin & 1.43 & 97 & Antibacterial \\
\hline Novobiocin sodium & 1.53 & 107 & Antibacterial \\
\hline Diphenyleneiodonium chloride & 1.97 & 101 & Antibacterial \\
\hline Phenylmercuric acetate & 2.77 & 99 & $\begin{array}{l}\text { Antifungal in } \\
\text { agriculture }\end{array}$ \\
\hline Sparfloxacin & 3.64 & 106 & Antibacterial \\
\hline Trovafloxacin mesylate & 4.18 & 102 & Antibacterial \\
\hline Erythromycin propionate & 4.35 & 110 & Antibacterial \\
\hline Enrofloxacin & 4.88 & 99 & Antibacterial \\
\hline Malachite green oxalate & 5.07 & 101 & $\begin{array}{l}\text { Antiseptic in } \\
\text { veterinary }\end{array}$ \\
\hline Marbofloxacin & 5.54 & 94 & Antibacterial \\
\hline Nitroxoline & 5.79 & 108 & Antibacterial \\
\hline Nitromersol & 5.90 & 94 & $\begin{array}{l}\text { Antiseptic and } \\
\text { disinfectant. }\end{array}$ \\
\hline Pifithrin-mu & 7.24 & 82 & $\begin{array}{l}\text { Antineoplastic } \\
\text { (p53 inhibitor) }\end{array}$ \\
\hline Chloroxine & 7.29 & 96 & Antibacterial \\
\hline 5-Fluorouracil & 7.29 & 79 & Antineoplastic \\
\hline Grepafloxacin $\mathrm{HCl}$ & 8.03 & 96 & Antibacterial \\
\hline Ticlatone & 8.45 & 88 & Antifungal \\
\hline Azithromycin dihydrate & 8.71 & 117 & Antibacterial \\
\hline Moxifloxacin $\mathrm{HCl}$ & 9.35 & 105 & Antibacterial \\
\hline Garenoxacin mesylate hydrate & 9.38 & 96 & Antibacterial \\
\hline Ofloxacin & 9.66 & 100 & Antibacterial \\
\hline Difloxacin $\mathrm{HCl}$ & 11.34 & 117 & Antibacterial \\
\hline Fusidic acid sodium & 11.34 & 124 & Antibacterial \\
\hline 6-Thioguanine & 11.98 & 77 & Antineoplastic \\
\hline Tosufloxacin toluenesulfonic acid & 14.54 & 76 & Antibacterial \\
\hline Dipyrithione & 16.86 & 111 & $\begin{array}{l}\text { Fungicidal and } \\
\text { bactericidal }\end{array}$ \\
\hline Nadifloxacin & 17.30 & 95 & Antibacterial \\
\hline Alatrofloxacin mesylate & 18.06 & 103 & Antibacterial \\
\hline Fluspirilene & 22.74 & 77 & Antipsychotic \\
\hline Bay 11-7082 & 23.57 & 99 & $\begin{array}{l}\text { Anti- } \\
\text { inflammatory }\end{array}$ \\
\hline Alexidine dihydrochloride & 29.02 & 111 & Antibacterial \\
\hline Disulfiram & 30.08 & 81 & $\begin{array}{l}\text { Chronic } \\
\text { alcoholism }\end{array}$ \\
\hline
\end{tabular}

therapies, three promising drug combination pairs with a synergistic effect against the drug resistant AB5075 strain were identified, including azithromycin/5-fluorouracil, colistin sulfate/fluspirilene, and colistin sulfate/Bay 11-7082. The synergistic effects of all three combination pairs were confirmed in a 96-well format assay. The $\mathrm{IC}_{50}$ values of known antibiotics was significantly reduced in the presence of the newly identified non-antibiotic agents. The $\mathrm{IC}_{50}$ value of azithromycin was reduced 6-fold, from 6.4 to $1.1 \mu \mathrm{M}$ in the presence of $1.8 \mu \mathrm{M}$ 5-fluorouracil (Figure 4A). Fluspirilene $(11.4 \mu \mathrm{M})$ and Bay 11-7082 $(11.8 \mu \mathrm{M})$ increased the inhibitory activity of colistin sulfate against the drug resistant AB5075 by 30- and 4-fold, respectively. The $\mathrm{IC}_{50}$ values of colistin were reduced from 0.22 to 0.0074 and $0.06 \mu \mathrm{M}$ in the presence of fluspirilene and Bay 11-7082, respectively (Figures 4B,C).

\section{DISCUSSION}

In this study, we describe the optimization and validation of a high throughput growth assay to measure the viability of a multidrug resistant Acinetobacter baumannii strain, AB5075. The 1,536-well format of the bacterial growth assay enabled the quick screening of thousands of compounds and drug combination sets with low reagent costs. The results from 25 known antibiotics revealed that AB5075 is resistant to most $\beta$ lactams, aminoglycosides, quinolones, and macrolides. The data agreed with these reported by Jacobs et al. (2014).

Seven non-antimicrobial agents, either approved drugs or in clinical trials, were identified in the compound screening campaign; these were confirmed as novel inhibitors of AB5075. For consideration of potential clinical applications of the newly identified antimicrobial activity of these compounds, the human plasma drug concentration levels should be higher than their IC $_{90}$ values or minimum inhibitory concentrations (MIC). Two drugs, among these seven confirmed antimicrobial agents, 5fluorouracil and 6-thioguanine, met this criterion; these two drugs are potent anticancer medications. Five-fluorouracil is a fluoropyrimidine and is a broad-spectrum anticancer agent, typically used as a first line chemotherapy agent for colorectal cancer (Longley et al., 2003). The standard treatment dose of 5-fluorouracil for cancer patients is $370 \mathrm{mg} / \mathrm{m}^{2}$ daily with a reported $\mathrm{C}_{\max }$ of $48.41 \mu \mathrm{g} / \mathrm{mL}$ (Bocci et al., 2000) which is higher than the $\mathrm{IC}_{90}(25.22 \mu \mathrm{g} / \mathrm{mL})$ we found in the AB5075 growth inhibition assay. It has dual inhibition mechanisms including functioning as an alternative substrate resulting in miscoding DNA and RNA and inhibiting thymidylate synthase. The broad spectrum antimicrobial activity of 5-fluorouracil was observed as early as 1985 (Bodet et al., 1985). Anti-microbial activity of 5 -fluorouracil has also been confirmed against $S$. aureus and S. epidermidis (Gieringer et al., 1986; Rangel-Vega et al., 2015). Mechanistic studies of antimicrobial activity are scant for 5-fluorouracil. However, the antimycotic mechanism of flucytosine suggests that 5-fluorouracil may share the same inhibitory mechanism against pathogens as it has against cancer (Vermes et al., 2000). Flucytosine, a prodrug, is converted into 5 -fluorouracil following cell uptake. 
TABLE 3 | Active and plasma concentration for non-antimicrobial indication candidates.

\begin{tabular}{|c|c|c|c|c|c|c|c|}
\hline \multirow[t]{2}{*}{ Drug name } & \multirow[t]{2}{*}{ Chemical structure } & \multirow[t]{2}{*}{$\mathrm{IC}_{50}(\mu \mathrm{M})$} & \multirow[t]{2}{*}{$\mathrm{IC}_{90}(\mu \mathrm{M})$} & \multirow{2}{*}{$\begin{array}{l}\text { Max } \\
\text { response }\end{array}$} & \multirow{2}{*}{$\begin{array}{l}\text { Primary } \\
\text { action }\end{array}$} & \multicolumn{2}{|c|}{$\mathrm{C}_{\max }$} \\
\hline & & & & & & $\mu \mathrm{g} / \mathrm{mL}$ & $\mu \mathbf{M}$ \\
\hline Auranofin & & 1.09 & 7.6 & 106 & Antirheumatic & $0.68^{\star}$ & 1 \\
\hline Pifithrin- $\mu$ & & 7.24 & 145.8 & 82 & $\begin{array}{l}\text { Antineoplastic } \\
\text { (p53 inhibitor) }\end{array}$ & $\mathrm{N} / \mathrm{A}$ & \\
\hline 6-Thioguanine & & 11.98 & 84.8 & 77 & Antineoplastic & $\begin{array}{l}15 \\
\text { Kovach et al., } \\
1986\end{array}$ & 87 \\
\hline Fluspirilene & & 22.74 & 71.7 & 77 & Antipsychotic & $\begin{array}{l}0.2 \times 10^{-3} \\
\text { Swart et al., } 1998\end{array}$ & $0.42 \times 10^{-3}$ \\
\hline Disulfiram & & 30.08 & 198.7 & 81 & $\begin{array}{l}\text { Chronic } \\
\text { alcoholism }\end{array}$ & $0.39 \times 10^{-3}$ & $\begin{array}{l}1.3 \times 10^{-3} \\
\text { Johansson, } 1992\end{array}$ \\
\hline
\end{tabular}

*http://www.prometheuslabs.com/Resources/PI/Ridaura.pdf.

Six-thioguanine is mainly used as a chemotherapy for myeloid leukemia and myeloid malignancies (Munshi et al., 2014). The recommended dose for acute non-lymphocytic leukemia patients is $2-3 \mathrm{mg} / \mathrm{kg}$ daily in an oral form as a single agent, or $75-200 \mathrm{mg} / \mathrm{m}^{2}$ daily when used as a combination therapy. At a dose of $65 \mathrm{mg} / \mathrm{m}^{2}$, the mean peak plasma concentration of 6-thioguanine ranges from 6-10 $\mu \mathrm{M}$ (Kovach et al., 1986). Although the plasma concentration of conventional dosage is lower than the IC $_{90}$, it has been reported that $87 \mu \mathrm{M}$ in human plasma has been achieved at a larger dose of $800-1200 \mathrm{mg} / \mathrm{m}^{2}$ (Presant et al., 1984; Kovach et al., 1986). As a guanosine structural analog, 6-thioguanine gets incorporated into DNA and RNA, blocking the biosynthesis of these two essential macromolecules. Additionally, 6-thioguanine hinders purine synthesis by inhibiting hypoxanthine phosphoribosyltransferase (Hprt). The bactericidal effect of 6-thioguanine against $S$. aureus and bacteriostatic effects against for E. coli and $S$. typhimurium were reported previously (Soo et al., 2016). Although there is no Hprt homolog in bacteria, the bacterial PRTases [Xanthine-guanine phosphoribosyltranferase (Gpt), hypoxanthine phosphoribosyltransferase $(\mathrm{Hpt})$ and adenine phosphoribosyltransferase (Apt)], show substrate binding-site conservation with Hprt, suggesting that these bacterial PRTases 
could be the molecular targets of 6-thioguanine (Wensing et al., 2014).

We have noticed that the majority of non-antimicrobial drugs we found in this repurposing screen are anticancer drugs. Because most anticancer drugs are cytotoxic and have serious side effects, it is a reasonable concern for application of anticancer drugs to treat infectious diseases. However, repositioning of anticancer drugs for infectious diseases has been reported (reviewed in Soo et al., 2016) despite the concerns of potential side effects. For example, miltefosine has been approved to treat leishmaniasis in 2014 (Berman, 2015). The application of gallium compounds to control $P$. aeruginosa in patients with cystic fibrosis is completed its phase II clinical trial (ClinicalTrials.gov ${ }^{1}$ ). Five-fluorouracil has been implemented as a coating agent for central venous catheters to prevent the bacterial colonization during treatment (Walz et al., 2008). Therefore, treatment needs to balance the therapeutic benefit with potential side effects before considering use of the anticancer drugs for the severe infectious diseases.

Azithromycin has pronounced activity against $H$. influenza as well as most gram-negative bacteria including Acinetobacter baumannii. Recently, two important findings support the potential of anti-multidrug resistant Acinetobacter baumannii for azithromycin. Azithromycin exhibited better efficacy in cell culture media and animal models than the canonical bacterial culture media (Lin et al., 2015) and it was effective against lipopolysaccharide deficient colistin-resistant strains (GarcíaQuintanilla et al., 2015). The AB5075 strain belongs to the latter case; in our study the minimal inhibitory concentration for $90 \%$ inhibition $\left(\mathrm{MIC}_{90}\right)$ of azithromycin was $8.5 \mu \mathrm{g} / \mathrm{mL}$, which is lower than the average from 15 clinical isolates of $64 \mu \mathrm{g} / \mathrm{mL}$ (Fernández Cuenca et al., 2003). However, using the breakpoint for $H$. influenza, $4 \mu \mathrm{g} / \mathrm{mL}$ from CLSI, AB5075 is not sensitive to azithromycin. The $\mathrm{MIC}_{90}$ value of azithromycin drops to a clinically attainable level through a synergistic effect with 5-fluorouracil in the two-drug combination therapy format.

The appearance of colistin resistance in Acinetobacter baumannii infections presents an urgent need for development of new therapeutics. Glycopeptides and hydrophobic compounds such as trimethoprim showed synergistic effects with colistin in an earlier study (Vidaillac et al., 2012). In this study, we identified two non-antimicrobial drugs which can resensitize the AB5075 strain to colistin, lowering the $\mathrm{MIC}_{90}$ of colistin to clinically achievable concentrations.

Fluspirilene belongs to the diphenylbutylpiperidine family, which were first-generation antipsychotics. The initial therapeutic dose is $2 \mathrm{mg}$ weekly followed by $1-10 \mathrm{mg}$ weekly injection for maintenance. The $\mathrm{C}_{\max }$ of fluspirilene at $2 \mathrm{mg}$ is about 200 pg/mL (Swart et al., 1998). Diphenylbutylpiperidines are dopamine D2 receptor antagonists and ameliorate the positive symptoms resulting from the hyperdopaminergic neurotransmission (Seeman, 1980). The finding of antifungal

${ }^{1}$ ClinicalTrials.gov. Available online at: https://clinicaltrials.gov/ct2/show/ NCT02354859

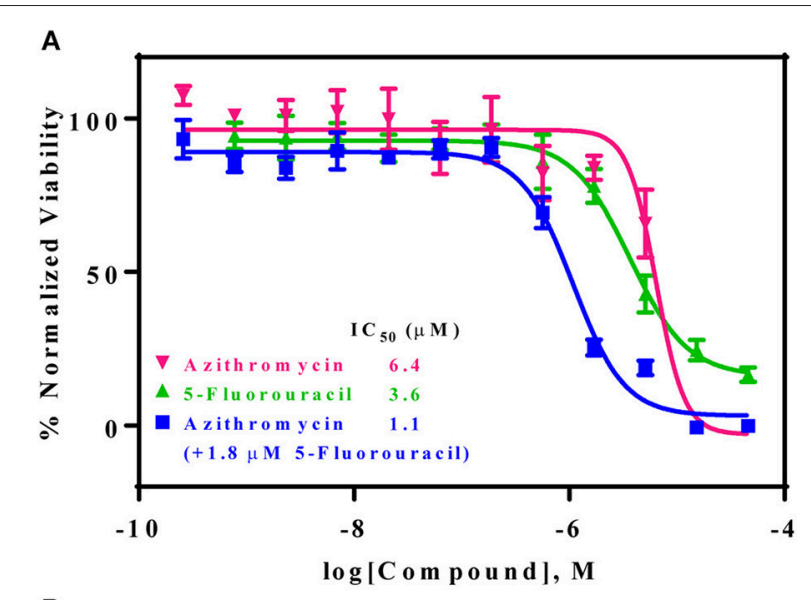

B

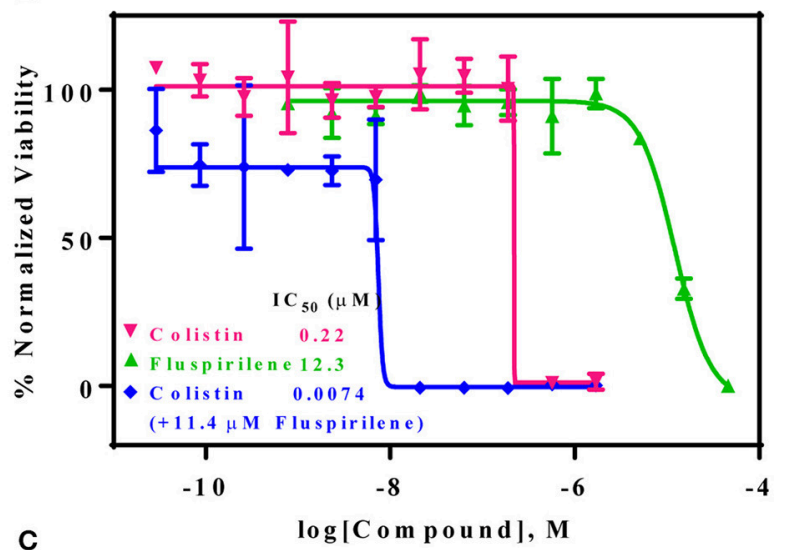

C $\log [\mathrm{Com}$ pound $], \mathrm{M}$

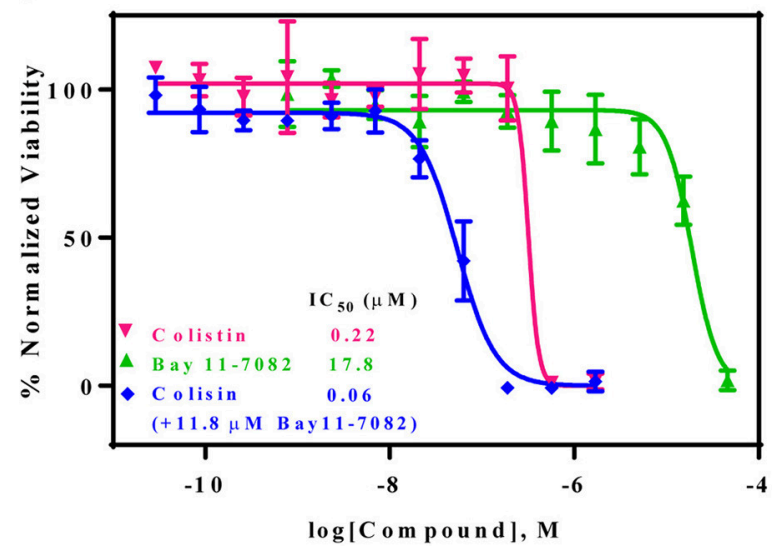

FIGURE 4 | Three non-conventional active compounds resensitize AB5075 to standard care antibiotics. AB5075 was treated with 5-fluorouracil, fluspirilene, or Bay 11-7082 combined with varying concentrations of azithromycin or colistin for $24 \mathrm{~h}$ at $37^{\circ} \mathrm{C}$ before detection of bacterial growth at $\mathrm{OD}_{600}$ (blue line). (A) In combination with $1.8 \mu \mathrm{M} 5$-fluorouracil the $\mathrm{IC}_{50}$ of azithromycin decreased $\sim 6$-fold $(6.4-1.1 \mu \mathrm{M})$. (B) In combination with 11.4 $\mu \mathrm{M}$ fluspirilene, the $\mathrm{IC}_{50}$ of colistin reduced $\sim 30$-fold $(0.22 \mu \mathrm{M}-7.4 \mathrm{nM})$. (C) In combination with $11.8 \mu \mathrm{M}$ Bay $11-7082$, the $\mathrm{IC}_{50}$ of colistin decreased $\sim 4$-fold $(0.22 \mu \mathrm{M}$ to $60 \mathrm{nM})$.

potential of fluspiriline supports a mechanistic study suggesting that fluspirilene likely inhibits the AB5075 growth by blockading the calcium-modulating protein, calmodulin (Butts et al., 2013). 
On the other hand, Bay 11-7082 is widely known as an $\mathrm{I} \kappa \mathrm{B}$ kinase (IKK) inhibitor. The molecular target of Bay 11-7082 remains unclear. From the chemical structural perspective, Bay 11-7082 is a phenyl vinyl sulfone related compound. The vinyl sulfone in conjugation with a nitrile group makes it a good Michael acceptor to interact with cysteine. The ability to inhibit cysteine proteases through an irreversible Michael addition were demonstrated in a recent study (Kerr et al., 2009). Notably, vinyl sulfone was identified as an anti-parasitic agent through this mechanism, suggesting cysteine proteases could be the molecular target of AB5075 inhibition (Kerr et al., 2009).

In conclusion, we have identified 43 approved drugs or drug candidates that significantly suppressed the growth of the multidrug resistant AB5075 strain including seven nonantimicrobial indication compounds. We also found three pairs of two drug combinations that exhibited synergistic effects with two known antibiotics against the AB5075 strain including azithromycin/5-fluorouracil, colistin sulfate/fluspirilene, and colistin sulfate/Bay 11-7082. These drug pairs are not contraindicated as only minor interactions between azithromycin and 5-fluorouracil have been reported which may be tolerable to patients. While these drug combination pairs may have the potential for clinical trials to treat multidrug resistant

\section{REFERENCES}

Berman, J. (2015). Miltefosine, an FDA-approved drug for the 'orphan disease', leishmaniasis. Expert Opin. Orphan Drugs 3, 727-735. doi: $10.1517 / 21678707.2015 .1039510$

Bocci, G., Danesi, R., Di Paolo, A. D., Innocenti, F., Allegrini, G., Falcone, A., et al. (2000). Comparative pharmacokinetic analysis of 5-fluorouracil and its major metabolite 5-fluoro-5,6-dihydrouracil after conventional and reduced test dose in cancer patients. Clin. Cancer Res. 6, 3032-3037.

Bodet, C. A., Jorgensen, J. H., and Drutz, D. J. (1985). Antibacterial activities of antineoplastic agents. Antimicrob. Agents Chemother. 28, 437-439. doi: 10.1128/AAC.28.3.437

Butts, A., DiDone, L., Koselny, K., Baxter, B. K., Chabrier-Rosello, Y., Wellington, M., et al. (2013). A repurposing approach identifies off-patent drugs with fungicidal Cryptococcal activity, a common structural chemotype, and pharmacological properties relevant to the treatment of Cryptococcosis. Eukaryot Cell 12, 278-287. doi: 10.1128/EC.00314-12

Demain, A. L. (1999). Pharmaceutically active secondary metabolites of microorganisms. Appl. Microbiol. Biotechnol. 52, 455-463. doi: $10.1007 / \mathrm{s} 002530051546$

Deng, M., Zhu, M. H., Li, J. J., Bi, S., Sheng, Z. K., Hu, F. S., et al. (2014). Molecular epidemiology and mechanisms of tigecycline resistance in clinical isolates of Acinetobacter baumannii from a Chinese university hospital. Antimicrob. Agents Chemother. 58, 297-303. doi: 10.1128/AAC.01727-13

Fernández Cuenca, F., Pascual, A., Martinez Martinez, L., and Perea, E. J. (2003). In vitro activity of azithromycin against clinical isolates of Acinetobacter baumannii. Rev. Esp. Quimioter. 16, 204-208.

Gallagher, L. A., Ramage, E., Weiss, E. J., Radey, M., Hayden, H. S., Held, K. G., et al. (2015). Resources for genetic and genomic analysis of emerging pathogen Acinetobacter baumannii. J. Bacteriol. 197, 2027-2035. doi: 10.1128/JB.00131-15

García-Quintanilla, M., Carretero-Ledesma, M., Moreno-Martinez, P., MartinPena, R., Pachon, J., and McConnell, M. J. (2015). Lipopolysaccharide loss produces partial colistin dependence and collateral sensitivity to azithromycin, rifampicin, and vancomycin in Acinetobacter baumannii. Int. J. Antimicrob. Agents 46, 696-702. doi: 10.1016/j.ijantimicag.2015.07.017
Acinetobacter baumannii infections, the other drugs found in this study may be useful for identification of new drugs scaffolds or new targets to combat this pathogen.

\section{AUTHOR CONTRIBUTIONS}

Y-SC, WS, and WZ conceived and designed the study. Y-SC, WS, and MX performed the experiments. MS performed the statistical analysis. MK and RS contributed materials. Y-SC wrote the first draft of the manuscript. All authors contributed to manuscript revision, read, and approved the submitted version.

\section{FUNDING}

This work was supported by the Intramural Research Program of the National Center for Advancing Translational Sciences (NCATS), National Institutes of Health.

\section{ACKNOWLEDGMENTS}

We thank the NCATS compound management group for their technical support and Dr. DeeAnn Visk, a medical writer and editor, for editing the manuscript.

Gieringer, J. H., Wenz, A. F., Just, H. M., and Daschner, F. D. (1986). Effect of 5 fluorouracil, mitoxantrone, methotrexate, and vincristine on the antibacterial activity of ceftriaxone, ceftazidime, cefotiam, piperacillin, and netilmicin. Chemotherapy 32, 418-424. doi: 10.1159/000238445

Huang, R., Southall, N., Wang, Y., Yasgar, A., Shinn, P., Jadhav, A., et al. (2011). The NCGC pharmaceutical collection: a comprehensive resource of clinically approved drugs enabling repurposing and chemical genomics. Sci. Transl. Med. 3:80ps16. doi: 10.1126/scitranslmed.3001862

Inglese, J., Auld, D. S., Jadhav, A., Johnson, R. L., Simeonov, A., Yasgar, A., et al. (2006). Quantitative high-throughput screening: a titration-based approach that efficiently identifies biological activities in large chemical libraries. Proc. Natl. Acad. Sci. U.S.A. 103, 11473-11478. doi: 10.1073/pnas.0604348103

Jacobs, A. C., Thompson, M. G., Black, C. C., Kessler, J. L., Clark, L. P., McQueary, C. N., et al. (2014). AB5075, a highly virulent isolate of Acinetobacter baumannii, as a model strain for the evaluation of pathogenesis and antimicrobial treatments. MBio 5, e01076-e01014. doi: 10.1128/mBio.01076-14

Johansson, B. (1992). A review of the pharmacokinetics and pharmacodynamics of disulfiram and its metabolites. Acta Psychiatr. Scand. Suppl. 369, 15-26. doi: 10.1111/j.1600-0447.1992.tb03310.x

Kerr, I. D., Lee, J. H., Farady, C. J., Marion, R., Rickert, M., Sajid, M., et al. (2009). Vinyl sulfones as antiparasitic agents and a structural basis for drug design. J. Biol. Chem. 284, 25697-25703. doi: 10.1074/jbc.M109.014340

Kovach, J. S., Rubin, J., Creagan, E. T., Schutt, A. J., Kvols, L. K., Svingen, P. A., et al. (1986). Phase I trial of parenteral 6-thioguanine given on 5 consecutive days. Cancer Res. 46, 5959-5962.

Lin, L., Nonejuie, P., Munguia, J., Hollands, A., Olson, J., Dam, Q., et al. (2015). Azithromycin synergizes with cationic antimicrobial peptides to exert bactericidal and therapeutic activity against highly multidrugresistant gram-negative bacterial pathogens. EBioMedicine 2, 690-698. doi: 10.1016/j.ebiom.2015.05.021

Longley, D. B., Harkin, D. P., and Johnston, P. G. (2003). 5-fluorouracil: mechanisms of action and clinical strategies. Nat. Rev. Cancer 3, 330-338. doi: $10.1038 / \mathrm{nrc10} 14$

Marinelli, F. (2009). Chapter 2. From microbial products to novel drugs that target a multitude of disease indications. Methods Enzymol. 458, 29-58. doi: 10.1016/S0076-6879(09)04802-2 
Munshi, P. N., Lubin, M., and Bertino, J. R. (2014). 6-thioguanine: a drug with unrealized potential for cancer therapy. Oncologist 19, 760-765. doi: 10.1634/theoncologist.2014-0178

Oikonomou, O., Sarrou, S., Papagiannitsis, C. C., Georgiadou, S., Mantzarlis, K., Zakynthinos, E., et al. (2015). Rapid dissemination of colistin and carbapenem resistant Acinetobacter baumannii in Central Greece: mechanisms of resistance, molecular identification and epidemiological data. BMC Infect. Dis. 15:559. doi: 10.1186/s12879-015-1297-x

Presant, C. A., Denes, A. E., Liu, C., and Bartolucci, A. A. (1984). Prospective randomized reappraisal of 5-fluorouracil in metastatic colorectal carcinoma. A comparative trial with 6-thioguanine. Cancer 53, 2610-2614. doi: 10.1002/ 1097-0142(19840615)53:12\&lt;2610::AID-CNCR2820531207\&gt;3.0.CO;2-9

Rangel-Vega, A., Bernstein, L. R., Mandujano-Tinoco, E. A., García-Contreras, S. J., and García-Contreras, R. (2015). Drug repurposing as an alternative for the treatment of recalcitrant bacterial infections. Front. Microbiol. 6:282. doi: $10.3389 /$ fmicb. 2015.00282

Seeman, P. (1980). Brain dopamine receptors. Pharmacol. Rev. 32, 229-313.

Soo, V. W., Kwan, B. W., Quezada, H., Castillo-Juarez, I., Perez-Eretza, B., Garcia-Contreras, S. J., et al. (2016). Repurposing of anticancer drugs for the treatment of bacterial infections. Curr. Top. Med. Chem. 17, 1157-1176. doi: $10.2174 / 1568026616666160930131737$

Sun, W., Weingarten, R. A., Xu, M., Southall, N., Dai, S., Shinn, P., et al. (2016). Rapid antimicrobial susceptibility test for identification of new therapeutics and drug combinations against multidrugresistant bacteria. Emerg. Microbes Infect. 5:e116. doi: 10.1038/emi. 2016.123

Swart, K. J., Sutherland, F. C., van Essen, G. H., Hundt, H. K., and Hundt, A. F. (1998). Determination of fluspirilene in human plasma by liquid chromatography-tandem mass spectrometry with electrospray ionisation. J. Chromatogr. A 828, 219-227. doi: 10.1016/S0021-9673(98) 00635-9

Vermes, A., Guchelaar, H. J., and Dankert, J. (2000). Flucytosine: a review of its pharmacology, clinical indications, pharmacokinetics, toxicity, and drug interactions. J. Antimicrob. Chemother. 46, 171-179. doi: 10.1093/jac/ 46.2.171
Vidaillac, C., Benichou, L., and Duval, R. E. (2012). In vitro synergy of colistin combinations against colistin-resistant Acinetobacter baumannii, Pseudomonas aeruginosa, and Klebsiella pneumoniae isolates. Antimicrob. Agents Chemother. 56, 4856-4861. doi: 10.1128/AAC.05996-11

Walz, J., Luber, J., Reyno, J., Stanford, G., Gitter, R., Longtine, K., et al. (2008). A multicenter randomized controlled clinical trial comparing central venous catheters impregnated with either 5 -fluorouracil or chlorhexidine/silver sulfadiazine in preventing catheter colonization. Crit. Care 12(Suppl. 2):P40. doi: 10.1186/cc6261

Wang, Y., Jadhav, A., Southal, N., Huang, R., and Nguyen, D. T. (2010). A grid algorithm for high throughput fitting of dose-response curve data. Curr. Chem. Genomics 4, 57-66. doi: 10.2174/1875397301004010057

Wensing, A., Gernold, M., Jock, S., Jansen, R., and Geider, K. (2014). Identification and genetics of 6-thioguanine secreted by Erwinia species and its interference with the growth of other bacteria. Mol. Genet. Genomics 289, 215-223. doi: 10.1007/s00438-013-0805-1

Xie, R., Zhang, X. D., Zhao, Q., Peng, B., and Zheng, J. (2018). Analysis of global prevalence of antibiotic resistance in Acinetobacter baumannii infections disclosed a faster increase in OECD countries. Emerg. Microbes Infect. 7:31. doi: 10.1038/s41426-018-0038-9

Zheng, W., Sun, W., and Simeonov, A. (2018). Drug repurposing screens and synergistic drug-combinations for infectious diseases. Br. J. Pharmacol. 175, 181-191. doi: 10.1111/bph.13895

Conflict of Interest Statement: The authors declare that the research was conducted in the absence of any commercial or financial relationships that could be construed as a potential conflict of interest.

Copyright (c) 2019 Cheng, Sun, Xu, Shen, Khraiwesh, Sciotti and Zheng. This is an open-access article distributed under the terms of the Creative Commons Attribution License (CC BY). The use, distribution or reproduction in other forums is permitted, provided the original author(s) and the copyright owner(s) are credited and that the original publication in this journal is cited, in accordance with accepted academic practice. No use, distribution or reproduction is permitted which does not comply with these terms. 